BULLETIN OF THE

AMERICAN MATHEMATICAL SOCIETY

Volume 77, Number 1, January 1971

\title{
THE ADAMS-NOVIKOV SPECTRAL SEQUENCE FOR THE SPHERES
}

\author{
BY RAPHAEL ZAHLER ${ }^{1}$ \\ Communicated by P. E. Thomas, June 17, 1970
}

The Adams spectral sequence has been an important tool in research on the stable homotopy of the spheres. In this note we outline new information about a variant of the Adams sequence which was introduced by Novikov [7]. We develop simplified techniques of computation which allow us to discover vanishing lines and periodicity near the edge of the $E_{2}$-term, interesting elements in $E_{2}^{2, *}$, and a counterexample to one of Novikov's conjectures. In this way we obtain independently the values of many low-dimensional stems up to group extension. The new methods stem from a deeper understanding of the Brown-Peterson cohomology theory, due largely to Quillen [8]; see also [4]. Details will appear elsewhere; or see [11].

When $p$ is odd, the $p$-primary part of the Novikov sequence behaves nicely in comparison with the ordinary Adams sequence. Computing the $E_{2}$-term seems to be as easy, and the Novikov sequence has many fewer nonzero differentials (in stems $\leqq 45$, at least, if $p=3$ ), and periodicity near the edge. The case $p=2$ is sharply different. Computing $E_{2}$ is more difficult. There are also hordes of nonzero differentials $d_{3}$, but they form a regular pattern, and no nonzero differentials outside the pattern have been found. Thus the diagram of $E_{4}$ $\left(=E_{\infty}\right.$ in dimensions $\left.\leqq 17\right)$ suggests a vanishing line for $E_{\infty}$ much lower than that of $E_{2}$ of the classical Adams spectral sequence [3].

It is a pleasure to thank Arunas Liulevicius, my thesis adviser, for his help. In particular, parts of the proofs of Proposition 1 and Theorem 7 are due to him. I am also grateful to many others for their suggestions, and especially to Frank Adams.

1. The spectral sequence. The construction of the classical Adams spectral sequence for the spheres [1] works equally well if the spec-

AMS 1970 subject classifications. Primary 55H15, 55G25, 55E45; Secondary $55 \mathrm{~B} 20$.

Key words and phrases. Stable homotopy of spheres, Adams spectral sequence, unitary cobordism, Brown-Peterson spectrum, projective resolution, Arf invariant, vanishing line, edge periodicity.

1 Most of the results announced in this paper were in the author's doctoral dissertation, written under an NSF graduate fellowship at the University of Chicago.

Copyright (C) 1971, American Mathematical Society 
trum $K\left(Z_{p}\right)$ representing ordinary cohomology is replaced by an arbitrary ring spectrum $X$. If $X$ satisfies certain conditions, the $E_{2}$-term of the resulting sequence will be isomorphic to

$$
\operatorname{Ext}_{A^{x}}\left(\Lambda^{x}, \Lambda^{x}\right)
$$

where $A^{x}=X^{*}(X)$ is the algebra of operations in $X$-cohomology theory and $\Lambda^{X}=\pi_{*}(X)$ is the coefficient ring. Novikov showed [7] that if $X=M U$ (the spectrum representing complex cobordism) this multiplicative spectral sequence converges to the stable homotopy ring $\pi_{*}^{s}$ :

$$
E_{\infty}^{s, t} \cong F^{s} \pi_{t-s}^{S} / F^{s+1} \pi_{t-s}^{S},
$$

where $F^{*}$ is a filtration of $\pi_{*}^{S}$. Furthermore, if $X^{\prime}=B P_{p}$, the BrownPeterson spectrum [4] for the prime $p$, the resulting spectral sequence $\left\{{ }_{p} E_{r},{ }_{p} d_{r}\right\}$ is exactly the $p$-primary part $\left\{E_{r} \otimes Q_{p}, d_{r} \otimes Q_{p}\right\}$ of the $M U$ spectral sequence $\left(Q_{p}\right.$ is the ring of rational numbers with denominators prime to $p$.)

Not much is known about the $M U$ spectral sequence, because even limited computations of $E_{2}$ have been difficult. This is regrettable, since what is known indicates that the Novikov sequence has certain a priori advantages over the usual one. The nonzero terms are sparse, for example: ${ }_{p} E_{2}^{s, t}=0$ if $t \neq 0 \bmod 2(p-1)$. Furthermore, almost all of the image of the $J$-homomorphism [2], [9] lies on the line $s=1$, in the following sense. According to Novikov, $E_{2}^{1,2 t}=Z_{m(t)}\left\langle\alpha_{t}\right\rangle$, a cyclic group with generator $\alpha_{t}$, isomorphic to the image of $J$ in dimension $2 t-1$ (isomorphic to $Z_{2}$ if $2 t-1 \equiv 5 \bmod 8$ ). There is a map $q_{1}: \pi_{n}^{3}$ $\rightarrow E_{2}^{1, n+1}$ such that an element of $E_{2}^{1, n+1}$ survives to $E_{\infty}$ iff it belongs to im $q_{1}$. Furthermore, if $\tilde{q}_{1}$ denotes the restriction of $q_{1}$ to im $J$, then [7, Chapters 10 and 11]

(1) if $n=8 k+1, E_{\infty}^{1, n+1}=E_{\infty}^{1, n+1}=Z_{2}$;

(2) if $n=8 k+3(k>0)$, then im $q_{1}=\operatorname{im} q_{1}$ has index 2 in $E_{2}^{1, n+1}$ $=Z_{m(4 k+2)}$, and $\tilde{q}_{1}$ has kernel $Z_{2}$; in fact, $d_{3} \alpha_{4 k+2}=h^{3} \alpha_{4 k} \neq 0$;

(3) if $n=8 k+5, E_{2}^{1, n+1}=Z_{2}$ does not survive to $E_{\infty}$; in fact, $d_{3} \alpha_{4 k+3}=h^{3} \alpha_{4 k+1} \neq 0$;

(4) if $n=8 k+7$, im $\tilde{q}_{1}=Z_{m(4 k+4)}=E_{2}^{1, n+1}=E_{\infty}^{1, n+1}$.

Here $h=\alpha_{1}$.

2. Quillen's algebra. Novikov knew that, given a prime $p$, the algebra $A^{B P}=B P^{*}(B P)$ was much simpler than $A^{M U} \otimes Q_{p}$, but he did not have complete information about $A^{B P}$. Later, Quillen [8] discovered an idempotent $\epsilon$, which split the spectrum $M U Q_{p}$ into a sum of suspensions of the spectrum $B P_{p}[4]$. Now 


$$
\pi_{*}(B P)=Q_{p}\left[k_{1}, k_{2}, \cdots\right], \quad H_{*}(B P)=Q_{p}\left[m_{1}, m_{2}, \cdots\right],
$$

with $\left|k_{i}\right|=-\left|m_{i}\right|=-2\left(p^{i}-1\right)$. We can take $m_{i}=\left(1 / p^{i}\right) h \epsilon\left[C P^{p^{i-1}}\right]$; the Hurewicz homomorphism $h$ is monic, and may be computed using Quillen's formal-group techniques [11] or standard methods. Thanks to the idempotent $\epsilon$, Quillen and Adams were able to write down explicit formulas for the Hopf-algebra structure of the algebra of operations $A^{B P}$ ( $=A$, for short).

First, there is a coalgebra $R$ of operations, free as a $Q_{p}$-module on generators $r_{E}$, where $E$ runs over all finitely nonzero sequences $\left(e_{1}, e_{2}, \cdots\right)$ of nonnegative integers and $\left|r_{E}\right|=2\left(\sum\left(p^{i}-1\right) e_{i}\right)$. The diagonal map is given by $\phi^{*} r_{E}=\sum_{E^{\prime}+E^{\prime \prime}=E} r_{E^{\prime}} \otimes r_{E^{\prime \prime}}$. Then $\Lambda^{\prime}$ $=\pi_{*}(B P)$ is an algebra over the coalgebra $R$, with action given (via the Hurewicz map) by $r_{E} m_{n}=m_{n-i}$ if $e_{i}=p^{n_{-i}}$ and all other $e_{j}$ are zero, and $r_{E} m_{n}=0$ otherwise. Moreover, multiplication by an element $\lambda$ of $\Lambda^{\prime}$ is also a $B P$-cohomology operation, and in fact every operation can be written as a (possibly infinite) sum $\sum \lambda_{i} r_{E_{i}}$ in which the degree of each $\lambda_{i} r_{E_{i}}$ is a constant independent of $i$. Unfortunately, the composition $r_{E} r_{F}$ of two operations in $R$ does not usually lie in $R$; however, it can be written uniquely as a finite sum $r_{E} r_{F}=\sum_{K} c_{K} r_{K}$ with $c_{K} \in \Lambda^{\prime}$, using the methods of [11] or those of [4]. This enables us to express compositions $\left(\lambda r_{E}\right)\left(\lambda^{\prime} r_{F}\right)$ in the form $\sum \lambda_{i} r_{E_{i}}$. Thus the algebra $A$ of all operations is the completed tensor product $\Lambda^{\prime} \hat{\otimes} R$.

Proposition 1. Let $\bar{\Lambda}$ be the two-sided ideal in $A$ generated by all elements of $\Lambda$ of negative degree. Let $a_{p} /\left(Q_{0}\right)$ be the algebra of reduced Steenrod pth powers [6]. Then there is an isomorphism $f: A / \bar{\Lambda}$ $\cong Q_{p} /\left(Q_{0}\right)$.

Proof. Let Th: $B P_{p} \rightarrow K\left(Z_{p}\right)$ be the $Z_{p}$ Thom class. Then

$$
\begin{array}{cc}
\bar{f}=\mathrm{Th}_{*}:[B P, B P] & \rightarrow\left[B P, K\left(Z_{p}\right)\right] \\
\| & \| \\
A & H^{*}\left(B P ; Z_{p}\right) \\
& a_{p} /\left(Q_{0}\right)
\end{array}
$$

satisfies

$$
\begin{aligned}
\bar{f}\left(k^{E} r_{F}\right) & =c\left(\rho^{F}\right), & & E=0[6] ; \\
& =0, & & \text { otherwise; }
\end{aligned}
$$

where $c$ is the canonical antiautomorphism. The map $\bar{f}$ induces the required $f$ on $A / \bar{\Lambda}$. 
A generator $r_{E}$ is indecomposable if it cannot be expressed as a finite sum $r_{E}=\sum \lambda_{i} R_{i} R_{i}{ }^{\prime}$, where $\lambda_{i} \in \Lambda^{\prime} ; R_{i}, R_{i}{ }^{\prime} \in R ;$ and $\left|R_{i}\right|$, $\left|R_{i}^{\prime}\right|>0$.

THEOREM 2. The generator $r_{E}$ of $R$ is indecomposable if and only if $E=\left(p^{i}, 0,0, \cdots\right), i \geqq 0$. Moreover, $\operatorname{pr}_{\left(p^{i}, 0,0, \ldots\right)}$ is decomposable.

The proof is obtained by noticing certain pleasant properties of the multiplication table for $R$ and applying them in the proper sequence.

3. Resolutions over $A$. To compute Ext we must construct resolutions over $A$, which seems difficult at first glance since $R$ is not an algebra, $A$ is not connected, and the ground ring $Q_{p}$ is not a field. The next proposition shows how to circumvent some of these difficulties. Define the filtrations $F^{s} \Lambda^{\prime}=\sum_{i \leq 2 s}\left(\Lambda^{\prime}\right)^{i}, F^{s} A=F^{s} \Lambda^{\prime} \hat{\otimes} A$, and $F^{s} M$ $=\left(F^{s} A\right) M$ if $M$ is an $A$-module. We have

$$
0 \rightarrow F^{1} M \stackrel{i}{\rightarrow} M \stackrel{j}{\rightarrow} \operatorname{cok} i \rightarrow 0 \text {. }
$$

Write $J M$ for cok $i$; then $J$ is easily made into a functor on the category of $A$-modules.

Proposition 3. There exist complexes

$$
C: \cdots \rightarrow C_{i} \stackrel{d_{i}}{\rightarrow} C_{i-1} \rightarrow \cdots \rightarrow C_{1} \stackrel{d_{1}}{\rightarrow} C_{0}=A \rightarrow \Lambda^{\prime} \rightarrow 0
$$

satisfying

(1) $C_{1}=\sum A u_{j}$ with $d_{1} u_{j}=r_{(p i, 0,0, \ldots) \text {; }}$

(2) $C_{i}=\prod_{j} A w_{j}^{(i)}$ is locally finitely generated as an $A$-module, $i>1$;

(3) $\operatorname{ker}\left(J d_{i}\right) \subset j\left(\mathrm{im} d_{i+1}\right)$ in $J C_{i}$ for all $i, n \geqq 0$.

$A$ ny such $C$ is an $A$-projective resolution of $\Lambda^{\prime}$.

The proof is straightforward. Notice that the infinite direct product $\prod A w_{j}^{(i)}$ is not necessarily free over $A$; it is projective, however. As a further aid to computation there is

LEMMA 4. If $\left\{C_{i}, d_{i}\right\}$ is any $A$-projective resolution of $\Lambda^{\prime}$, write $C_{i}^{*}=\operatorname{Hom}_{A}^{*}\left(C_{i}, \Lambda^{\prime}\right), d_{i}^{*}=\operatorname{Hom}_{A}^{*}\left(d_{i}, \Lambda^{\prime}\right)$. Then

$$
\operatorname{Ext}_{\Lambda}^{s, t}\left(\Lambda^{\prime}, \Lambda^{\prime}\right)=\operatorname{Tors}\left(\operatorname{cok}\left(d_{s}^{*}\right)^{t}\right), \quad(s, t) \neq(0,0) .
$$

Proof. This follows from the fact that $\operatorname{Ext}_{A^{U}}^{s, t}$ is finite for $(s, t)$ $\neq(0,0)[7$, Corollary 2.1].

Thus in determining Ext we need know just the boundaries, and not the cycles too. In fact we can even work over $Z_{p^{f}}$ for suitable $f$. 
Now we can prove

Proposition 5. Ext ${ }^{0, t}=0$ unless $t=0 ; \operatorname{Ext}^{0,0}=Z$.

THEOREM 6. Ext $\mathrm{t}^{2, t}$ contains a direct summand isomorphic to $Z_{p}$ for $t=2 p^{i}(p-1)(i \geqq 1)$ and $t=2\left(p^{i}+1\right)(p-1)(i>1)$.

Theorem 7. For $p=2$, the element of $\mathrm{Ext}^{2,2^{i}}$ found in Theorem 6 maps to the Arf-invariant element $h_{i}^{2}$ of the classical Adams spectral seguence [5].

Proof. Apply the Thom map (Proposition 1) to a suitable $A$-resolution.

Proposition 8. The two-primary part ${ }_{2} \mathrm{Ext}^{\mathrm{s}, t}$ has the following "edge" values:

$$
\begin{aligned}
{ }_{2} \mathrm{Ext}^{n, 2(n+k)} & =0, & & k<0 ; \\
& =Z_{2}, & & k=0, n \geqq 1\left(\text { generated by } h^{n}\right) ; \\
& =0, & & k=1, n \geqq 2 ; \\
& =Z_{2}, & & \left.2 \leqq k \leqq 5, n \geqq 4 \text { (generated by } h^{n-1} \alpha_{k+1}\right) .
\end{aligned}
$$

Further computations of the additive structure of ${ }_{2} \mathrm{Ext}^{*}, *$ in low dimensions are given in Figure 1. Thanks to Proposition 8, the first three nonzero Novikov differentials $d_{3} \alpha_{i}=h^{3} \alpha_{i-1}, i=3,6,7$, give rise to infinite towers of nonzero $d_{3}$ 's. Moreover, every other differential in the range $t-s \leqq 17$ must be zero for dimensional reasons. Finally, ${ }_{2} E_{\infty}$ has a vanishing line considerably lower than that of the $E_{\infty}$-term of the classical Adams spectral sequence in this range of dimensions. We conjecture that the preceding four sentences are also true without restriction on the dimensions.

Similar computations for $p=3$ disclose striking edge properties like Proposition 8 , but many fewer differentials. Contrary to Novikov's conjecture [7], there is a nonzero differential $d_{5}: E_{2}^{2.36} \rightarrow E_{2}^{7.40}$ for $p=3$. This differential, whose existence is inferred from Toda's result [10], also gives rise to an infinite family of nonzero differentials. It is encouraging that there is only one nonzero differential in the range $t-s \leqq 40$, as compared to 17 in the classical 3-primary Adams spectral sequence.

\section{REFERENCES}

1. J. F. Adams, On the structure and applications of the Steenrod algebra, Comment. Math. Helv. 32 (1958), 180-214. MR 20 \#2711.

2. - On the groups $J(X)$. II, Topology 3 (1965), 137-173; IV, ibid. 5 (1966), 21-71. MR 33 \#6626; 6628. 
3. - A periodicity theorem in homological algebra, Proc. Cambridge Philos. Soc. 62 (1966), 365-377. MR 33 \#2696.

4. - Q Quillen's work on formal group laws and complex cobordism, University of Chicago Lecture Notes Series, 1970.

5. W. Browder, The Kervaire invariant of framed manifolds and its generalization, Ann. of Math. (2) 90 (1969), 157-186.

6. J. W. Milnor, The Steenrod algebra and its dual, Ann. of Math. (2) 67 (1958), 150-171. MR $20 \# 6092$.

7. S. P. Novikov, The method of algebraic topology from the point of view of cobordism theory, Izv. Akad. Nauk SSSR Ser. Mat. 31 (1967), 855-951=Math. USSR Izv. 1 (1967), 827-913. MR 36 \#4561.

8. D. Quillen, On the formal group laws of unoriented and complex cobordism theory, Bull. Amer. Math. Soc. 75 (1969), 1293-1298.

9. - The Adams conjecture (to appear).

10. H. Toda, An important relation in homotopy groups of spheres, Proc. Japan Acad. 43 (1967), 839-842. MR 37 \#5872.

11. R. Zahler, The Adams-Novikov spectral sequence for the spheres, Thesis, University of Chicago, Chicago, Ill., 1970.

University of California, Berkeley, California 94720

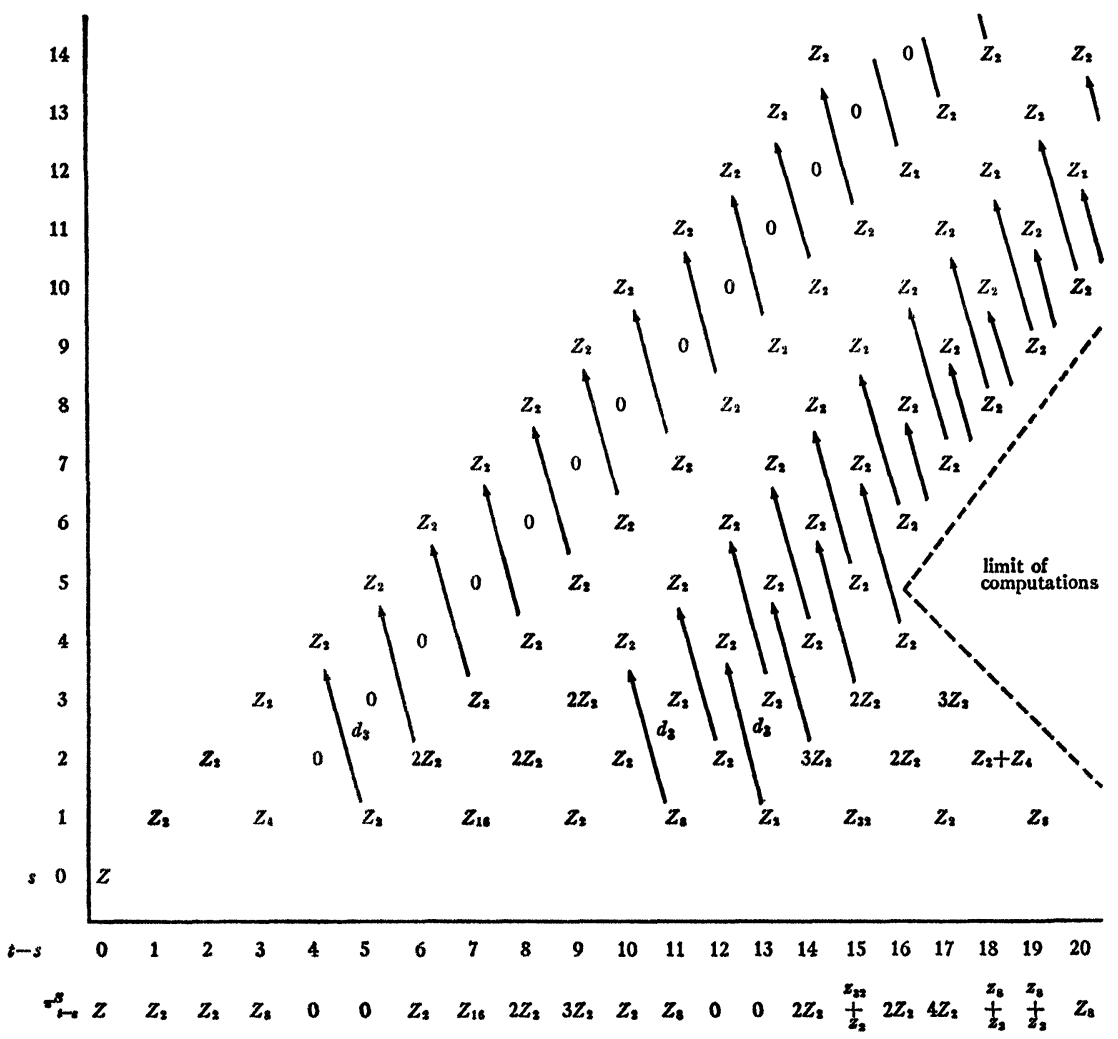

FIGURE 1. ${ }_{2} \mathrm{Ext}^{8, t}$ for the Novikov sequence. 